\title{
Outcomes of Cryoballoon Ablation in High- and Low-Volume Atrial Fibrillation Ablation Centres: A Russian Pilot Survey
}

\author{
Evgeny N. Mikhaylov, ${ }^{1,2}$ Dmitry S. Lebedev, ${ }^{1}$ Evgeny A. Pokushalov, ${ }^{3}$ \\ Karapet V. Davtyan, ${ }^{4}$ Eduard A. Ivanitskii, ${ }^{5}$ Anatoly A. Nechepurenko, ${ }^{6}$ \\ Alexey Ya. Kosonogov, ${ }^{7}$ Grigory V. Kolunin, ${ }^{8}$ Igor A. Morozov, ${ }^{9}$ \\ Sergey A. Termosesov, ${ }^{10}$ Evgeny B. Maykov, ${ }^{11}$ Dmitry N. Khomutinin, ${ }^{12}$ \\ Sergey A. Eremin, ${ }^{13}$ Igor M. Mayorov, ${ }^{14}$ Alexander B. Romanov, ${ }^{3}$ \\ Vitaliy V. Shabanov, ${ }^{3}$ Victoria Shatakhtsyan, ${ }^{4}$ Viktor Tsivkovskii, ${ }^{5}$ \\ Amiran Sh. Revishvili, ${ }^{15}$ and Evgeny V. Shlyakhto ${ }^{16}$
}

${ }^{1}$ Arrhythmia Department, Federal Almazov North-West Medical Research Centre, Saint Petersburg 197341, Russia

${ }^{2}$ Neuromodulation Unit, Federal Almazov North-West Medical Research Centre, Saint Petersburg 197341, Russia

${ }^{3}$ Center of Interventional Cardiology, State Research Institute of Circulation Pathology, Novosibirsk 630055, Russia

${ }^{4}$ National Research Center for Preventive Medicine, Moscow 101990, Russia

${ }^{5}$ Federal Center for Cardiovascular Surgery, Krasnoyarsk 660020, Russia

${ }^{6}$ Federal Center for Cardiovascular Surgery, Astrakhan 414011, Russia

${ }^{7}$ Electrophysiology Department, City Hospital \#5, Nizhny Novgorod 603005, Russia

${ }^{8}$ Electrophysiology Department, Tyumen Cardiology Center, Tyumen 625026, Russia

${ }^{9}$ Electrophysiology Department, Research Institute of Cardiology, Saratov 410028, Russia

${ }^{10}$ Cardiovascular Surgery Department, City Hospital \#12, Moscow 115516, Russia

${ }^{11}$ Department of Clinical Electrophysiology, Russian Cardiology Research and Production Center, Moscow 121552, Russia

${ }^{12}$ Tyumen Regional Hospital \#1, Tyumen 625032, Russia

${ }^{13}$ Republican Clinical Hospital of the Republic of Tatarstan, Kazan 420064, Russia

${ }^{14}$ Botkin City Hospital, Moscow 125284, Russia

${ }^{15}$ Bakulev Scientific Center of Cardiovascular Surgery, Moscow 121552, Russia

${ }^{16}$ Institute of Heart and Vessels, Federal Almazov North-West Medical Research Centre, Saint Petersburg 197341, Russia

Correspondence should be addressed to Evgeny N. Mikhaylov; e.mikhaylov@almazovcentre.ru

Received 18 August 2015; Accepted 27 October 2015

Academic Editor: Laurent Pison

Copyright (C) 2015 Evgeny N. Mikhaylov et al. This is an open access article distributed under the Creative Commons Attribution License, which permits unrestricted use, distribution, and reproduction in any medium, provided the original work is properly cited.

Purpose. The results of cryoballoon ablation (CBA) procedure have been mainly derived from studies conducted in experienced atrial fibrillation (AF) ablation centres. Here, we report on CBA efficacy and complications resulting from real practice of this procedure at both high- and low-volume centres. Methods. Among 62 Russian centres performing AF ablation, 15 (24\%) used CBA technology for pulmonary vein isolation. The centres were asked to provide a detailed description of all CBA procedures performed and complications, if encountered. Results. Thirteen sites completed interviews on all CBAs in their centres ( $>95 \%$ of CBAs in Russia). Six sites were high-volume AF ablation ( $>100 \mathrm{AF}$ cases/year) centres, and 7 were low-volume AF ablation. There was no statistical difference in arrhythmia-free rates between high- and low-volume centres (64.6 versus $60.8 \%$ at 6 months). Major complications developed in $1.5 \%$ of patients and were equally distributed between high- and low-volume centres. Minor procedurerelated events were encountered in $8 \%$ of patients and were more prevalent in high-volume centres. Total event and vascular access site event rates were higher in women than in men. Conclusions. CBA has an acceptable efficacy profile in real practice. In less experienced AF ablation centres, the major complication rate is equal to that in high-volume centres. 


\section{Introduction}

Cryoballoon ablation (CBA) for pulmonary vein (PV) isolation has become a treatment used worldwide for patients with atrial fibrillation (AF). As with other "single-shot device" technologies, CBA aims to simplify and shorten the PV ablation procedure in patients with recurrent arrhythmia [1]. The number of electrophysiology centres is growing continuously, and some sites may not be well experienced in complex procedures when they launch AF ablation programmes [2]. It is not yet well understood which PV isolation technology is best advised for centres just starting to offer interventional AF management, since different energy sources and types of ablation have different learning curves and complication rates [3].

We sought to investigate whether CBA started in centres not previously experienced in AF ablation procedures might be safe and effective. Therefore, the aim of this study was to describe the techniques and results of CBA PV isolation in the Russian Federation and to compare AF ablation results between high- and low-volume AF ablation centres beginning to use this cryoablation technology.

\section{Methods}

According to the information obtained from a national electrophysiology centres registry, among 62 centres performing AF ablation between 2010 and 2014 in the Russian Federation, 23 sites used catheter cryotechnology. Of those centres, 15 (24\%) performed cryoballoon PV isolation. These centres were contacted and asked to participate in a national survey. Fourteen centres responded, and a general questionnaire was provided to centres' representatives. Thirteen (87\%) centres returned a fully completed questionnaire. In a second step, an additional questionnaire was sent to centres that had reported procedure-related adverse events.

The study was approved by the Federal North-West Medical Research Centre ethics committee. The study was conducted under the auspices of the Russian Society of Cardiology and the Russian Scientific Society of Clinical Electrophysiology, Arrhythmology and Cardiac Pacing.

2.1. General Questionnaire. The general questionnaire consisted of 8 sections and 74 questions in total (Supplementary Table 1 in Supplementary Material available online at http://dx.doi.org/10.1155/2015/591603): centre experience in catheter ablation (7 questions); centre experience in cryoballoon ablation (8 questions); cryoballoon ablation technique (28 questions); patient follow-up methods (8 questions); periprocedural anticoagulation (6 questions); CBA efficacy (3 questions); procedure-related events and complications (9 questions); recurrence specifications (5 questions).

In order to investigate a mean number of AF procedures per year and to classify the volume of AF ablation centres, responders were required to report measures collected during the 2 most recent years. Investigation of clinical outcomes (arrhythmia-free rate) was based on a 6-month follow-up period in the centres with an outpatient control programme.
Additionally, the 12-month arrhythmia-free rate was analyzed separately, when available.

2.2. Additional Questionnaire upon Procedure-Related Events. Ten centres reporting procedure-related events were asked to provide additional detailed information regarding patient characteristics and further management (Supplementary Table 2). Additional questions were addressed to centres to clarify ambiguous responses and in order to obtain left atrial sizes, PV isolation verification techniques and successes, and so forth. All centres provided full responses to additional questions.

Major complications were classified as procedure-related events, requiring additional interventions and/or prolonging hospitalization. Other conditions were classified as procedure-related minor events: vascular access site problems (minor groin haematoma, etc.), transient phrenic nerve paresis/dysfunction with restoration during the index procedure, pericardial effusion without consequence, and so forth.

2.3. Statistical Analysis. Continuous variables were expressed as mean \pm standard deviation and were compared using the $t$-test, if their distribution did not deviate significantly from the normal distribution (tested with the KolmogorovSmirnov test). If significant deviation from the normal distribution was found, continuous variables were expressed as the median within the interquartile range (IQR) and were compared using nonparametric tests (Mann-Whitney $U$ tests and Fisher's exact test). Categorical variables were expressed as percentages and numbers. Weighted average percentages were provided for percentages taken from different totals. Percentages were compared using the "difference between proportions" function. A 2 -tailed $p<0.05$ indicated statistical significance. Analysis was performed using Statistica 6.0 software (StatSoft Inc., Tulsa, OK, USA).

\section{Results}

3.1. Centres' Characteristics. Average experience in catheter ablation among the 13 participating centres was $9.8 \pm 5.7$ years, with AF ablation programmes started on average $6.2 \pm$ 3.4 years ago. The median number of ablation procedures was 2149 (IQR: 1330-4010) per centre.

More than 100 AF procedures per year were performed in 6 centres (median 202.6; IQR 142.3-493.4 AF cases per year), and they were classified as higher-volume AF ablation sites according to the 2012 HRS/EHRA/ECAS Expert Consensus Statement, which declared that better AF ablation outcomes are seen in centres with $>100$ cases [3]. The other 7 centres performed < 100 (median 25.7; IQR: 13-89.4) AF cases per year and were classified as lower-volume AF ablation sites (Table 1). Additionally, safety and efficacy were calculated separately for the 3 most experienced centres, performing $>400$ AF ablations per year.

Furthermore, in order to investigate CBA outcomes in centres with different experience in all arrhythmia types ablation (not only AF), the sites were divided into lowablation experience (1st quartile, $\leq 1330$ ablations; 4 centres), 
TABLE 1: AF ablation centres' characteristics.

\begin{tabular}{|c|c|c|c|c|c|}
\hline Centre code & Total number of catheter ablations & Total number of CBAs & Total AF ablations & AF ablations/year* & High AF volume center \\
\hline 1 & 12001 & 109 & 5232 & 1011 & + \\
\hline 2 & 1596 & 71 & 568 & 202 & + \\
\hline 3 & 4298 & 69 & 1532 & 411 & + \\
\hline 4 & 3416 & 55 & 353 & 123 & + \\
\hline 5 & 2149 & 33 & 284 & 89 & - \\
\hline 6 & 7434 & 27 & 2364 & 575 & + \\
\hline 7 & 4010 & 24 & 1237 & 161 & + \\
\hline 8 & 2445 & 18 & 52 & 15 & - \\
\hline 9 & 235 & 13 & 40 & 25 & - \\
\hline 10 & 1330 & 13 & 16 & 6 & - \\
\hline 11 & 912 & 10 & 217 & 60 & - \\
\hline 12 & 1750 & 8 & 43 & 3 & - \\
\hline 13 & 128 & 7 & 26 & 12 & - \\
\hline Total & 41704 & 457 & 11964 & 2693 & $46 \%$ \\
\hline
\end{tabular}

${ }^{*}$ Calculated for the 2 last years; AF: atrial fibrillation; CBA: cryoballoon ablation.

medium-ablation experience ( $>1330$ and $\leq 4010$ procedures; 5 centres), and high-ablation experience (3rd quartile, $>4010$ procedures; 4 centres).

The median proportion of CBA procedures among all $\mathrm{AF}$ ablations was $12.5 \%$ (IQR: $4.5-26.9 \%$ ) per centre.

The number of radiofrequency (RF) AF ablation procedures increased in 2013/2014 by $15 \%$ per month, while the absolute number of CBA procedures increased by $214 \%$.

Two nonresponding centres were low-volume AF ablation sites. One centre performed 6 CBA procedures (data from the centre) and the other less than 20 (data from the company). Therefore, the survey data included 457 procedures, representing $>95 \%$ of all CBA cases performed in the country.

3.2. Patient Characteristics. A single CBA procedure was performed in 457 patients (56.3\% males; mean age $54.8 \pm 5.4$ years). In 428 (94\%) patients, AF was paroxysmal, and in $29(6 \%)$ it was persistent. In all patients, arrhythmia was refractory to at least one antiarrhythmic drug.

3.3. Ablation Procedure Characteristics. Ultrasound guidance for transseptal access was routinely used in 10 centres but used only in certain cases in 1 centre (5 transoesophageal, 4 intracardiac, and 2 either transoesophageal or intracardiac echo). Only 2 centres adopted intracardiac ultrasound guidance for controlling balloon positioning within PV ostia.

Double transseptal punctures were used in 7 centres and single punctures in 6 . An additional circular diagnostic catheter was used in 8 centres. The number of transseptal punctures was strongly dependent on the use of the integrated Achieve (Medtronic, Minneapolis, MN, USA) circular catheter. Sites that adopted this catheter switched to one transseptal access.

All centres used the Arctic Front balloon (Medtronic, Minneapolis, MN, USA), since the second-generation balloon was not available in the Russian Federation until 2015. The balloon catheter was introduced into the left atrium via a 14-F transseptal steerable sheath (FlexCath, Medtronic, Minneapolis, MN, USA). Among the 13 centres, 12 used only $28 \mathrm{~mm}$ balloons, while 1 low-volume centre adopted exclusively $23 \mathrm{~mm}$ balloons (aiming to obtain a higher occlusion rate of all PVs). All centres reported that at least two freeze cycles of $300 \mathrm{~s}$ in duration were delivered to each PV.

In all participating centres, the endpoint of the CBA procedure was complete electrical disconnection of all PVs. RF touch-up applications, in order to complete PV isolation, were reported by 4 centres (in $5.1 \%(0-23 \%)$ of patients); operators preferred RF applications due to faster performance and lower cost of this technology in comparison with cryo touch-ups. No linear ablation in the left atrium was performed. Concomitant right atrial isthmus RF ablation was performed in patients with documented typical flutter. Bidirectional block in the PVs was the endpoint of the CBA in 9 centres, whereas in 4 centres operators aimed to achieve entrance block as the endpoint. According to the prespecified endpoint, isolation of all PVs was obtained in $89.3 \%$ of patients. A nonisolated PV was almost exclusively the right inferior PV.

The mean procedure time was $155.7 \pm 35.7 \mathrm{~min}$ and the mean fluoroscopy time was $27.7 \pm 10.2 \mathrm{~min}$ among all centres. Four high-volume centres provided fluoroscopy and total procedure times for the first 20 and all subsequent procedures: $36.0 \pm 14.5$ versus $31.0 \pm 15.8 \mathrm{~min}$ and $191.3 \pm 29.5$ versus $158.5 \pm 18.9 \mathrm{~min}$, respectively $(p>0.05)$.

Implementation of the Achieve circular catheter was associated with a statistically significant decrease in the mean fluoroscopy and total procedure times: $29.8 \pm 10.8$ versus $26.3 \pm 10.4 \mathrm{~min}$ and $165.4 \pm 22.0$ versus $141.2 \pm 37.6 \mathrm{~min}$, correspondingly, $p<0.05$.

Periprocedural anticoagulation strategies were mixed in 12 centres; operators used bridge anticoagulation with low molecular weight heparin in 10 centres and uninterrupted Warfarin in 6. Periprocedural anticoagulation with novel oral anticoagulants (NOACs) was reported by 11 centres: Dabigatran was skipped $12-48$ hours before the procedure 
and Rivaroxaban 12-24 hours before the procedure. One centre used an uninterrupted Warfarin strategy only.

3.4. Patient Follow-Up. Median hospital stay was 3 (1-6) days (IQR: 3-4). Regular patient checks were performed in 11 centres. Recurrence documentation was carried out by resting ECG registration and 24-hour Holter monitoring (every 1-6 months) in 11 sites; subcutaneous loop recorders were implanted in 2 centres (in $60 \%$ and $13 \%$ of patients in these centres). Regular personal visits to outpatient units were performed in 7 centres, and 9 centres additionally contacted patients by phone (every 3,6 , or 12 months).

Ten centres reported 6-month arrhythmia-free rates of $63.9 \%$ after paroxysmal AF CBA off antiarrhythmics, after a 3-month blanking period. Persistent AF CBA was performed in 2 centres with arrhythmia-free rates of $50 \%$ and $66.7 \%$ at 6 months off antiarrhythmics.

Six centres provided 1-year follow-up data with a mean arrhythmia-free rate of $64.1 \%$ (50-75.8\%) off antiarrhythmic drugs. One of the two centres, which used implantable loop recorders for recurrence documentation, reported a $66 \%$ arrhythmia-free rate at one year (off antiarrhythmics).

Regular supraventricular tachycardia as a recurrence was encountered in 18 (4\%) patients. In $11(2.4 \%)$ patients, it was finally diagnosed as typical atrial flutter. Left atrial tachycardia (AT) was documented in $6(1.3 \%)$ patients, and in $1(0.2 \%)$ patient it was a macroreentrant AT.

Additionally, sites were asked to report the number of redo cases following CBA. Twelve centres provided data within 12 months of follow-up: a redo procedure was performed in 25 patients ( $7.1 \%$ of patients out of 12 centres); RF PV reisolation was carried out in all cases. The centres reported that in redo cases they used the point-by-point $\mathrm{RF}$ technique instead of CBA due to the lower cost of the procedure and possible mapping necessity for non-PV tachycardias.

3.5. Procedure-Related Events. Major complications developed in seven $(1.5 \%)$ patients. Cardiac tamponade was encountered in 4 patients, all of which were managed by percutaneous drainage without further sequelae. Ischaemic stroke occurred in 2 patients: 1 patient completely recovered, while the other patient had partial permanent disability. Persistent phrenic nerve palsy ( $>6$ months) was reported in 1 patient. No atrioesophageal fistulas or deaths within 60 days were reported.

Minor procedure-related events were encountered in 37 (8\%) patients and included transient phrenic nerve paresis/dysfunction, haemoptysis, and pericardial effusion (Table 2). In the centre which adopted the $23 \mathrm{~mm}$ balloon, one transient phrenic nerve paresis was seen. In 5 patients with haemoptysis, symptoms resolved spontaneously within 2 days. Additional questions were addressed to operators regarding this complication. It was noted that the minimum temperature during ablation in these patients was from -53 to $-67^{\circ} \mathrm{C}$. No specific reason for this adverse event was identified; a $28 \mathrm{~mm}$ balloon was used in these patients.

The total procedure-related event rate was higher in women than in men $(12 \%$ versus $4.9 \%$; $p<0.05)$, mainly due to the higher prevalence of vascular access site minor events in females ( $6 \%$ in women versus $0 \%$ in men; $p<0.05)$.

3.6. Cryoballoon Ablation in High-versus Low-Volume AF Ablation Centres. In compliance with the criteria of centre volume, two groups had significant differences in the number of total catheter-ablation procedures and number of AF procedures (Table 2). On the other hand, there was only a borderline difference between the durations of high- and low- volume centres' AF ablation programmes ( $p=0.05)$. The mean proportion of CBAs among all AF ablation cases was higher in low-volume than in the high-volume centres. A higher proportion of male patients were present in the high-volume centres. There was no statistical difference in the routine use of ultrasound guidance during the procedure or in mean fluoroscopy and total procedure times. Isolation of all PVs was more frequently achieved in high-volume centres (Table 2), and the right inferior PV was the only nonisolated vein in some patients in low-volume centres.

Mid-term (6 months) freedom from any arrhythmia was comparable between high- and low-volume AF ablation centres.

Major complication rates were similar in high- and lowvolume centres $(1.4 \%$ versus $2.0 \%, p>0.05)$. Minor procedure-related events were more frequently seen in highvolume centres than in low-volume AF ablation centres $(9.9 \%$ versus $1.0 \%, p=0.001)$. Due to the prevalence of minor events, the total number of all adverse events was higher in the high-volume centres. However, there were no differences in specific conditions between the groups (Table 2).

When calculated in the 3 highest-volume centres $(>400$ AF cases per year; 205 CBA procedures in total), CBA success and major complication rates did not differ from those in the low-volume centres (68.9\% and 2.4\%, resp., $p>$ $0.05)$. However, minor adverse events were reported more frequently $(8.3 \% ; p=0.04)$.

3.7. Cryoballoon Ablation Outcomes in Centres with Different Experience in All Arrhythmia Types Ablation. When centres were classified according to experience in ablation of all arrhythmia types, it was found that minor procedure-related events were still more frequently seen in high-experienced centres, but in comparison only with medium-experienced centres (Table 3). There were no differences in arrhythmiafree rates and major complication rates between high- and low-experienced centres.

\section{Discussion}

In low-volume AF ablation centres, where operators have experience in simple ablation procedures, CBA is a safe procedure with success rates comparable to those obtained in higher-volume AF centres. Major complications of CBA are infrequently encountered in real practice and rarely lead to permanent disability. Our results underline that CBA has a fast and reproducible learning curve in both high- and lowvolume AF ablation centres. 
TABLE 2: Characteristics of procedure results in high- and low-volume AF ablation centres.

\begin{tabular}{|c|c|c|c|c|}
\hline Parameter & Overall & $\begin{array}{l}\text { High-volume } \mathrm{AF} \\
\text { ablation centres, } \\
\quad N=6\end{array}$ & $\begin{array}{l}\text { Low-volume } \mathrm{AF} \\
\text { ablation centres, } \\
\quad N=7\end{array}$ & $p$ \\
\hline Mean experience in catheter ablation, years & $9.8 \pm 5.7$ & $9.7 \pm 4.2$ & $10.9 \pm 6.3$ & 0.7 \\
\hline Median number of catheter-ablation procedures & 2149 (IQR: 1330-4010) & $\begin{array}{c}4010 \text { (IQR: } \\
2782.5-5866)\end{array}$ & $\begin{array}{l}1750 \text { (IQR: } \\
912-2445)\end{array}$ & 0.008 \\
\hline Median number of catheter-ablation procedures per year & 266.7 (IQR: 97.1-569.3) & $\begin{array}{c}569.3 \text { (IQR: } \\
266.7-842.8)\end{array}$ & $\begin{array}{l}114 \text { (IQR: } \\
\text { 78.3-267.3) }\end{array}$ & 0.005 \\
\hline Median experience in AF ablation, years & 5 (IQR: 4-8) & 6 (IQR: 5-10.5) & 5 (IQR: 4-8) & 0.05 \\
\hline Median number of AF ablation procedures & 284 (IQR: 43-1237) & $\begin{array}{c}1237 \text { (IQR: } \\
460.5-1948.5)\end{array}$ & 52 (IQR: 40-284) & 0.001 \\
\hline Median number of AF ablation procedures per year & 89.4 (IQR: $15-202.6)$ & $\begin{array}{c}202.6 \text { (IQR: } \\
142.3-493.4)\end{array}$ & 25.7 (IQR: 13-89.4) & 0.001 \\
\hline Mean number of CBAs & $35.2 \pm 29.0$ & $59.1 \pm 31.7$ & $14.6 \pm 8.9$ & 0.004 \\
\hline Proportion of CBA among all AF cases & 12.5\% (IQR: 4.5-26.9) & 4.5\% (IQR: 2-12) & $\begin{array}{c}18.6 \% \text { (IQR: } \\
4.6-13)\end{array}$ & 0.01 \\
\hline Mean age, years & $54.8 \pm 5.4$ & $53.6 \pm 2.3$ & $55.9 \pm 7.1$ & 0.46 \\
\hline Gender, $\%$ of males & $56.3 \%$ & $62.8 \%$ & $50.8 \%$ & 0.002 \\
\hline Left atrial diameter, $\mathrm{mm}$ & 41.9 & 41.8 & 42.1 & 0.33 \\
\hline Routine echo guidance, number of centers & 10 & 4 & 6 & 1.05 \\
\hline \multicolumn{5}{|l|}{ Periprocedural anticoagulation strategy, centers } \\
\hline Uninterrupted Warfarin & 6 & 2 & 4 & 0.59 \\
\hline Bridge anticoagulation & 10 & 5 & 5 & 1.0 \\
\hline NOACs & 11 & 5 & 6 & 1.0 \\
\hline Fluoroscopy time, min & $27.7 \pm 10.2$ & $24.6 \pm 9.9$ & $33.8 \pm 9.5$ & 0.34 \\
\hline Procedure time, $\min$ & $155.7 \pm 35.7$ & $154.5 \pm 28.4$ & $156.8 \pm 44.8$ & 0.91 \\
\hline RF touch-up applications, centers & 4 & 3 & 1 & 0.19 \\
\hline Isolation of all PVs, $\%$ of patients & $89.3 \%$ & $97 \%$ & $79.4 \%$ & $<0.001$ \\
\hline Arrhythmia-free rate at 6 months & $63.9 \%$ & $64.6 \%$ & $60.8 \%$ & 0.96 \\
\hline Major complications & $7(1.5 \%)$ & $5(1.4 \%)$ & $2(2.0 \%)$ & 0.66 \\
\hline Tamponade & $4(0.87 \%)$ & $3(0.84 \%)$ & $1(0.98 \%)$ & 1.0 \\
\hline Stroke & $2(0.4 \%)$ & $2(0.56 \%)$ & 0 & 1.0 \\
\hline Persistent phrenic nerve palsy & $1(0.2 \%)$ & 0 & $1(0.98 \%)$ & 1.0 \\
\hline Minor procedure-related events & $37(8 \%)$ & $35(9.9 \%)$ & $2(2.0 \%)$ & 0.007 \\
\hline Pericardial effusion & $1(0.2 \%)$ & $1(0.28 \%)$ & 0 & 1.0 \\
\hline Transient phrenic nerve paresis & $23(5 \%)$ & $21(5.9 \%)$ & 2 & 0.08 \\
\hline Vascular complications & $8(1.8 \%)$ & $8(2.2 \%)$ & 0 & 0.21 \\
\hline Arteriovenous fistula & $3(0.65 \%)$ & $3(0.84 \%)$ & 0 & 1.0 \\
\hline Femoral artery pseudoaneurysm & $5(1 \%)$ & $5(1.4 \%)$ & 0 & 0.59 \\
\hline Transient hemoptysis & $5(1 \%)$ & $5(1.4 \%)$ & 0 & 0.59 \\
\hline Total procedure-related events & $44(9.6 \%)$ & $40(11.3 \%)$ & $4(3.9 \%)$ & 0.03 \\
\hline
\end{tabular}

AF: atrial fibrillation; CBA: cryoballoon ablation; IQR: interquartile range; NOACs: novel oral anticoagulants; RF: radiofrequency.

4.1. Cryoballoon Ablation Procedure Characteristics. As shown in this study, the number of CBA procedures is growing, especially in centres that have recently started AF ablation programmes. A similar increase in the number of CBAs has been reported in a German AF ablation registry [4].

In accordance with the results of previous studies, CBA has a short learning curve and usually about $20-50$ procedures are required to reach a plateau $[5,6]$. In this study, no significant decline in fluoroscopy and procedure times was observed after the first $20 \mathrm{CBA}$ procedures, most likely due to the limited data provided. However, implementation of an integrated diagnostic circular catheter was associated with a significant reduction in fluoroscopy and procedure durations and these findings echo previous reports [7].

Fluoroscopy and total procedure times were shorter in our study than those in previous reports $[1,8,9]$. It is suggested that previous personal experience in simpler 
TABLE 3: CBA procedure outcomes in centres with low-, medium-, and high-catheter-ablation experience (all types of arrhythmia ablations).

\begin{tabular}{|c|c|c|c|c|c|c|}
\hline Parameter & $\begin{array}{l}\text { I } \\
\text { Low ablation experience ( } 4 \\
\text { centres) }\end{array}$ & $\begin{array}{l}\text { II } \\
\text { Medium ablation } \\
\text { experience }(5 \text { centres })\end{array}$ & $\begin{array}{l}\text { III } \\
\text { High ablation experience }(4 \\
\text { centres) }\end{array}$ & $\begin{array}{c}\text { Between } \\
\text { I and II }\end{array}$ & $\begin{array}{c}p \\
\text { Between } \\
\text { II and III }\end{array}$ & $\begin{array}{l}\text { Between } \\
\text { I and III }\end{array}$ \\
\hline $\begin{array}{l}\text { Median number of } \\
\text { catheter-ablation } \\
\text { procedures }\end{array}$ & 573 (IQR: 208-1016) & $\begin{array}{l}2445 \text { (IQR: } \\
2149-3416)\end{array}$ & 5866 (IQR: 3622-8575) & 0.69 & 0.01 & 0.007 \\
\hline $\begin{array}{l}\text { Median number of } \\
\text { AF ablation } \\
\text { procedures }\end{array}$ & 33 (IQR: 23-84) & 284 (IQR: 52-353) & 1948 (IQR: 1291-3079) & 0.02 & 0.02 & $<0.001$ \\
\hline $\begin{array}{l}\text { Mean number of } \\
\text { CBAs }\end{array}$ & 11 (IQR: 9-13) & 24 (IRQ: 18-33) & $69 \pm 33.5$ & 0.01 & 0.25 & 0.002 \\
\hline $\begin{array}{l}\text { Total number } \\
\text { of CBAs }\end{array}$ & 43 & 138 & 276 & NA & NA & NA \\
\hline $\begin{array}{l}\text { Fluoroscopy time, } \\
\text { min }\end{array}$ & $30.8 \pm 11.7$ & $29.9 \pm 3.0$ & $25.0 \pm 12.3$ & 0.08 & 0.05 & 0.97 \\
\hline Procedure time, min & $165.0 \pm 41.0$ & $159.2 \pm 43.4$ & $142.8 \pm 27.6$ & 0.92 & 0.48 & 0.53 \\
\hline $\begin{array}{l}\text { Arrhythmia-free rate } \\
\text { at } 6 \text { months, } \%\end{array}$ & $66.6 \%$ & $56.2 \%$ & $67.5 \%$ & 0.11 & 0.05 & 0.87 \\
\hline $\begin{array}{l}\text { Major complications, } \\
N(\%)\end{array}$ & $1(2.3 \%)$ & $1(0.7 \%)$ & $5(1.8 \%)$ & 0.42 & 0.67 & 0.58 \\
\hline $\begin{array}{l}\text { Minor } \\
\text { procedure-related } \\
\text { events, } N(\%)\end{array}$ & $2(4.6 \%)$ & $4(2.9 \%)$ & $26(9.4 \%)$ & 0.63 & 0.015 & 0.49 \\
\hline $\begin{array}{l}\text { Total } \\
\text { procedure-related } \\
\text { events, } N(\%)\end{array}$ & $3(6.9 \%)$ & $5(3.6 \%)$ & $31(11.2 \%)$ & 0.40 & 0.009 & 0.60 \\
\hline
\end{tabular}

AF: atrial fibrillation; CBA: cryoballoon ablation; IQR: interquartile range.

ablation procedures might play an important role in this result.

Another interesting finding was the low rate of additional touch-up applications required for PV isolation, especially in the low-volume centres. Although the intended endpoint of CBA was complete PV isolation in all centres, the right inferior PV was sometimes left nonisolated by operators with less experience in RF left atrial ablation, instead of performing aggressive attempts with additional equipment to complete isolation. Nevertheless, when comparing recurrence rates in the high- and low-volume sites, this does not seem influencing the mid-term results significantly, suggesting that the right inferior PV might play a minor role in arrhythmogenesis.

Periprocedural anticoagulation strategy was mixed in the majority of centres and depended on what kind of anticoagulant was used in patients prior to ablation. We did not receive information on specific anticoagulation strategies in patients with cardiac tamponade or vascular adverse events. Previous studies have suggested that there is no significant difference between the use of uninterrupted Warfarin and the use of NOACs [10]. Several randomized studies addressing this issue are underway.

4.2. Cryoballoon Ablation Efficacy. The mid-term arrhythmia-free rate (at 6 and 12 months of follow-up) in our survey was slightly lower when compared with previous AF ablation surveys and studies $[1,4,8,11-13]$. We suggest that this could be related to the fact that all participating centres were relatively inexperienced in the use of the cryotechnology. Moreover, only the first-generation balloon was used in all centres.

In our study, two centres reported success rates of persistent AF CBA. This form of AF was present only in $6 \%$ of patients treated; therefore, we are not able to show any reliable comparison with the group of paroxysmal AF. The results of CBA in this group were promising; however, we should appreciate the small size of this patient subgroup.

Regular supraventricular tachycardia as a recurrence has been found in $4 \%$ of patients and was a macroreentrant left AT in only 1 patient. The low incidence of AT and reentrant arrhythmia after CBA was in accordance with previously published reports $[8,14]$.

\subsection{Ablation Results Using First- and Second-Generation} Cryoballoons. Importantly, our survey results reflect experience with the first-generation cryoballoon. Currently, a second-generation cryoballoon is available in many countries and several studies have compared the efficacy of the firstand second-generation devices. These reports show that the arrhythmia-free rate after ablation using the newer balloon yields significantly better results ranging around $80-84 \%$ [1517].

It has been also shown that a single application approach using the newer balloon reduces cryoablation and fluoroscopy times while achieving excellent results [18, 19]. All 
these findings indicate that the newer device is more powerful when compared to the first-generation balloon.

The main idea of this study was to compare AF CBA in different volume centres, and the cryoablation procedure performed with either the first- or second-generation balloons is still a "one-shot" device technology for achieving $\mathrm{PV}$ isolation. Moreover, we believe that the performance of the ablation procedure was more reproducible among the centres, since all of them used the same first-generation balloon.

4.4. Procedure-Related Events. It should be acknowledged that, in the low-volume AF ablation centres, personal operators' experience in simple ablation procedures was considerable and some operators had performed >1000 ablation procedures for supraventricular and ventricular tachyarrhythmias before launching AF ablation programmes. Therefore, the results of this study should be interpreted in light of previous experience of operators regarding vascular access and intracardiac manipulations.

Cardiac tamponade occurred in $0.87 \%$ of cases. Considering the fact that about a quarter of CBAs were performed in low-volume AF ablation centres, this number appears low [20]. Since $61 \%$ of centres used ultrasound guidance for transseptal access, it is possible that this significantly decreased the risk of cardiac perforation. Moreover, one would expect that the risk of cardiac perforation during CBA might be lower than during RF ablation, since cryolesion cannot induce tissue overheating and wall disruption. In a study of about 35,000 AF ablation procedures, it was found that a considerable number of left atrial perforations occurred during RF energy delivery in the left atrium [20].

In patients with haemoptysis, no reasons for its development were identified and questions regarding the aetiology of this condition remained unanswered. Several reports of haemoptysis after CBA procedures have been published before and the authors suggested several potential causes, including lung tissue injury by distal balloon inflation, direct injury by a guidewire, or parenchymal pulmonary infarction due to acute PV occlusion [21]. We noted that the minimum ablation temperature achieved in these patients was low $(-53$ to $-67^{\circ} \mathrm{C}$ ), and this is in accordance with previous reports [22].

Our study showed low incidence of persistent $(>6$ months) phrenic nerve palsy. We believe that this was related to the almost exclusive use of a first-generation $28 \mathrm{~mm}$ balloon and close supervision of phrenic nerve function during right $\mathrm{PV}$ ablation by all operators. Previous studies have identified an association between a higher proportion of phrenic nerve damage with a smaller balloon size and the second-generation device $[5,15]$.

Gastroparesis has been earlier suggested as one of the underreported AF ablation complications [23]. It should be acknowledged that no cases of gastroparesis were documented in our survey.

A higher proportion of procedure-related minor events were found in higher-volume AF ablation centres. This finding is further confirmed by evaluation of CBA outcomes in centres with different experience in all arrhythmia types ablation. This can be partly explained by the fact that, in the lower-volume AF ablation centres, venous access was performed by well experienced vascular puncture operators. The higher-volume centres were mainly academic teaching centres, where preparations for ablation procedures were carried out by younger fellow physicians.

Our findings show that female gender patients might be associated with higher incidence of minor procedure-related events, mainly vascular access site complications. It is difficult to explain such prevalence in females, since we had no access to individual clinical data of patients without adverse events. Theoretically, a higher proportion of vascular access site problems in women might be associated with more increased body mass index or more variable femoral vein course. No gender difference was found in major complications; however, this could be explained by a limited number of cases for such analysis.

4.5. Clinical Implications. It seems that very experienced operators mainly prefer RF catheter ablation with an acceptable efficacy rate and low incidence of major complications. However, RF PV isolation requires substantial operator experience, with previous work having been closely supervised, and the failure rates and number of complications during the initial stages of gaining experience are high $[2,3]$. In this regard, CBA may be advocated for low-volume AF ablation centres, or centres starting their AF ablation programme, as a safe and equally effective procedure in properly selected patients.

\section{Study Limitations}

There are several limitations that may influence the results of this study. The study data are mainly derived from questionnaires, and the study is a survey in nature. A limited number of centres should be acknowledged. However, this was a national survey and the participating sites represented $>86 \%$ of all sites performing CBA in the country. Another limitation is that there was a difference in arrhythmia recurrence detection methods (Holter monitoring, implantable loop recorder interrogation) and a difference in time intervals between follow-up visits in the centres.

We had no access to detailed clinical information regarding patients without complications; therefore, we were not able to analyze predictors associated with a favourable outcome or complications.

Since there were more trainees in the high-volume centres, this might have also affected long term results in these centres. However, it is known that trainees were closely supervised by very experienced operators during the main stage of the procedure.

\section{Conclusion}

In conclusion, CBA can be safely performed in low-volume $\mathrm{AF}$ ablation centres without compromising efficacy. 


\section{Conflict of Interests}

Dr. E. Mikhaylov has received speaker honoraria from Medtronic. Drs. E. Pokushalov, E. Ivanitskii, A. Nechepurenko, G. Kolunin, A. Romanov, and V. Shabanov have received consulting fees/honoraria from Medtronic. The other authors report no conflict of interests.

\section{Acknowledgments}

The authors thank Drs. Evgeny B. Kropotkin, Alexandr V. Pavlov, Igor M. Khamnagadaev, Ilya L. Ilyich, and Anna V. Patsouk for their contribution to data collection.

\section{References}

[1] D. L. Packer, R. C. Kowal, K. R. Wheelan et al., "Cryoballoon ablation of pulmonary veins for paroxysmal atrial fibrillation: first results of the North American arctic front (STOP AF) pivotal trial," Journal of the American College of Cardiology, vol. 61, no. 16, pp. 1713-1723, 2013.

[2] A. Deshmukh, N. J. Patel, S. Pant et al., "In-hospital complications associated with catheter ablation of atrial fibrillation in the United States between 2000 and 2010: analysis of 93801 procedures," Circulation, vol. 128, no. 19, pp. 2104-2112, 2013.

[3] H. Calkins, K. H. Kuck, R. Cappato et al., "2012 HRS/EHRA/ ECAS expert consensus statement on catheter and surgical ablation of atrial fibrillation: recommendations for patient selection, procedural techniques, patient management and follow-up, definitions, endpoints, and research trial design: a report of the Heart Rhythm Society (HRS) Task Force on Catheter and Surgical Ablation of Atrial Fibrillation," Europace, vol. 14, no. 4, pp. 528-606, 2012.

[4] M. Schmidt, U. Dorwarth, D. Andresen et al., "Cryoballoon versus $\mathrm{RF}$ ablation in paroxysmal atrial fibrillation: results from the German Ablation Registry," Journal of Cardiovascular Electrophysiology, vol. 25, no. 1, pp. 1-7, 2014.

[5] Y. Van Belle, P. Janse, M. J. Rivero-Ayerza et al., "Pulmonary vein isolation using an occluding cryoballoon for circumferential ablation: feasibility, complications, and short-term outcome," European Heart Journal, vol. 28, no. 18, pp. 2231-2237, 2007.

[6] M. Wójcik, A. Berkowitsch, H. Greis et al., "Learning curve in cryoballoon ablation of atrial fibrillation-eight-year experience," Circulation Journal, vol. 78, no. 7, pp. 1612-1618, 2014.

[7] M. Peyrol, P. Sbragia, A. Quatre et al., "Reduction of procedure duration and radiation exposure with a dedicated inner lumen mapping catheter during pulmonary vein cryoablation," Pacing and Clinical Electrophysiology, vol. 36, no. 1, pp. 24-30, 2013.

[8] T. Neumann, J. Vogt, B. Schumacher et al., "Circumferential pulmonary vein isolation with the cryoballoon technique results from a prospective 3-center study," Journal of the American College of Cardiology, vol. 52, no. 4, pp. 273-278, 2008.

[9] D. S. Lebedev, E. N. Mikhaylov, V. S. Orshanskaya et al., "Cryoballoon ablation for pulmonary vein isolation to treat atrial fibrillation: first experience in Russian Federation," Kardiologiya, vol. 52, no. 4, pp. 38-48, 2012.

[10] A. A. Bin Abdulhak, A. R. Khan, I. M. Tleyjeh et al., "Safety and efficacy of interrupted dabigatran for peri-procedural anticoagulation in catheter ablation of atrial fibrillation: a systematic review and meta-analysis," Europace, vol. 15, no. 10, pp. 14121420, 2013.
[11] M. Khne, Y. Suter, D. Altmann et al., "Cryoballoon versus radiofrequency catheter ablation of paroxysmal atrial fibrillation: biomarkers of myocardial injury, recurrence rates, and pulmonary vein reconnection patterns," Heart Rhythm, vol. 7, no. 12, pp. 1770-1776, 2010.

[12] R. Cappato, H. Calkins, S.-A. Chen et al., "Updated worldwide survey on the methods, efficacy, and safety of catheter ablation for human atrial fibrillation," Circulation: Arrhythmia and Electrophysiology, vol. 3, no. 1, pp. 32-38, 2010.

[13] E. Arbelo, J. Brugada, G. Hindricks et al., "The atrial fibrillation ablation pilot study: a European Survey on methodology and results of catheter ablation for atrial fibrillation conducted by the European Heart Rhythm Association," European Heart Journal, vol. 35, no. 22, pp. 1466-1478, 2014.

[14] E. N. Mikhaylov, R. Bhagwandien, P. A. Janse, D. A. M. J. Theuns, and T. Szili-Torok, "Regular atrial tachycardias developing after cryoballoon pulmonary vein isolation: incidence, characteristics, and predictors," Europace, vol. 15, no. 12, pp. 1710-1717, 2013.

[15] G. Ciconte, L. Ottaviano, C. de Asmundis et al., "Pulmonary vein isolation as index procedure for persistent atrial fibrillation: one-year clinical outcome after ablation using the secondgeneration cryoballoon," Heart Rhythm, vol. 12, no. 1, pp. 60-66, 2015.

[16] A. Fürnkranz, S. Bordignon, D. Dugo et al., "Improved 1year clinical success rate of pulmonary vein isolation with the second-generation cryoballoon in patients with paroxysmal atrial fibrillation," Journal of Cardiovascular Electrophysiology, vol. 25, no. 8, pp. 840-844, 2014.

[17] F. Squara, A. Zhao, E. Marijon et al., "Comparison between radiofrequency with contact force-sensing and secondgeneration cryoballoon for paroxysmal atrial fibrillation catheter ablation: a multicentre European evaluation," Europace, vol. 17, no. 5, pp. 718-724, 2015.

[18] E. Wissner, C. Heeger, H. Grahn et al., "One-year clinical success of a 'no-bonus' freeze protocol using the secondgeneration $28 \mathrm{~mm}$ cryoballoon for pulmonary vein isolation," Europace, vol. 17, no. 8, pp. 1236-1240, 2015.

[19] G. Ciconte, C. de Asmundis, J. Sieira et al., "Single 3-minute freeze for second-generation cryoballoon ablation: one-year follow-up after pulmonary vein isolation," Heart Rhythm, vol. 12, no. 4, pp. 673-680, 2015.

[20] Y. Michowitz, M. Rahkovich, H. Oral et al., "Effects of sex on the incidence of cardiac tamponade after catheter ablation of atrial fibrillation: results from a worldwide survey in 34943 atrial fibrillation ablation procedures," Circulation: Arrhythmia and Electrophysiology, vol. 7, no. 2, pp. 274-280, 2014.

[21] R. Bhagwandien, Y. Van Belle, N. de Groot, and L. Jordaens, "Hemoptysis after pulmonary vein isolation with a cryoballoon: an analysis of the potential etiology," Journal of Cardiovascular Electrophysiology, vol. 22, no. 9, pp. 1067-1069, 2011.

[22] N. Kumar, C. Timmermans, M. Das et al., "Hemoptysis after cryoablation for atrial fibrillation: truth or just a myth?" Chest, vol. 146, no. 5, pp. e173-e175, 2014.

[23] T. Aksu, S. Golcuk, T. E. Guler, K. Yalin, and I. Erden, "Gastroparesis as a complication of atrial fibrillation ablation," The American Journal of Cardiology, vol. 116, no. 1, pp. 92-97, 2015. 


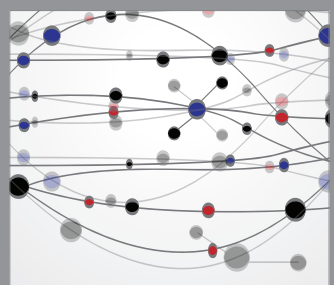

The Scientific World Journal
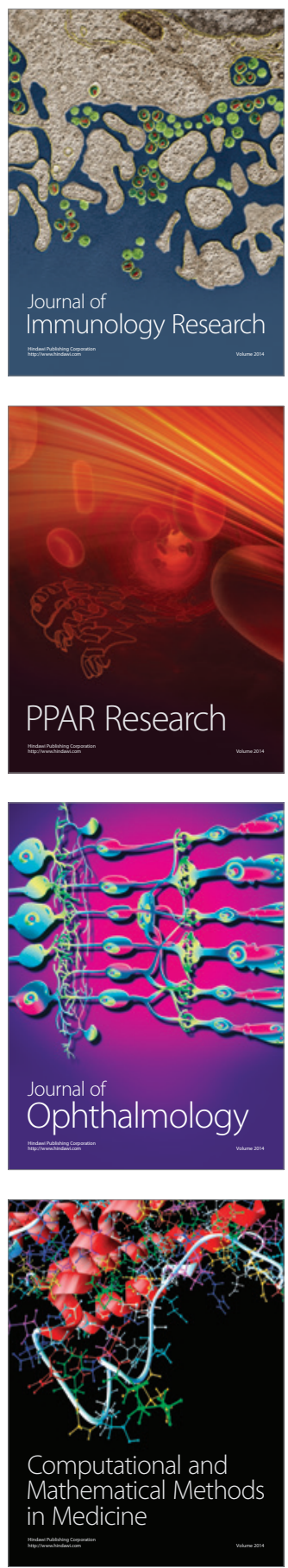

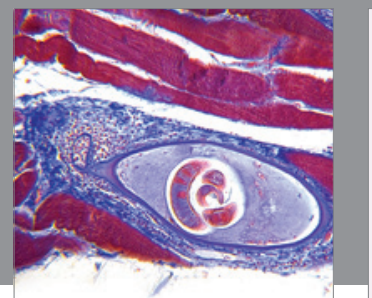

Gastroenterology

Research and Practice
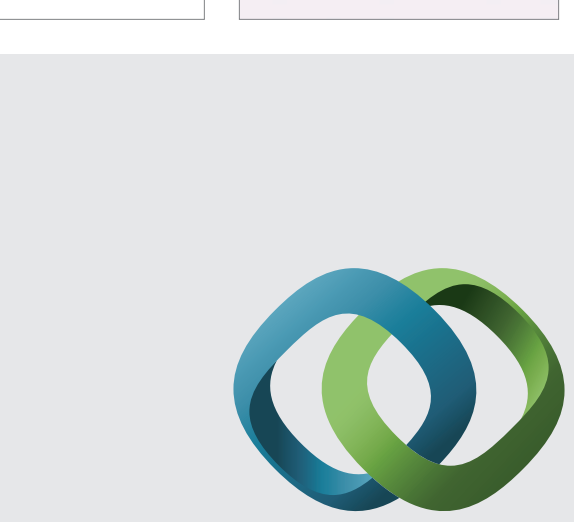

\section{Hindawi}

Submit your manuscripts at

http://www.hindawi.com
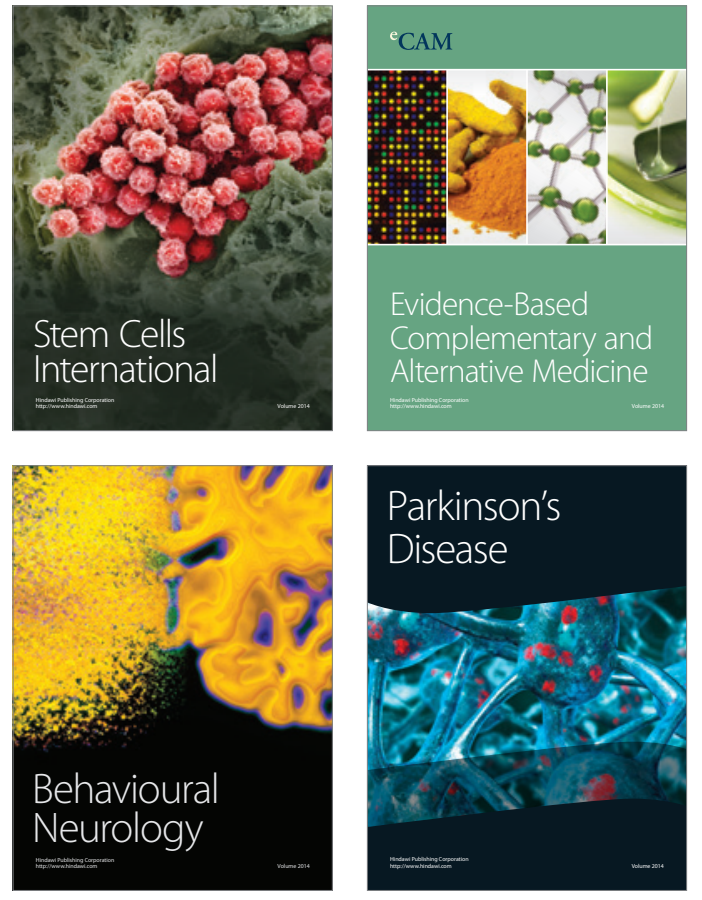
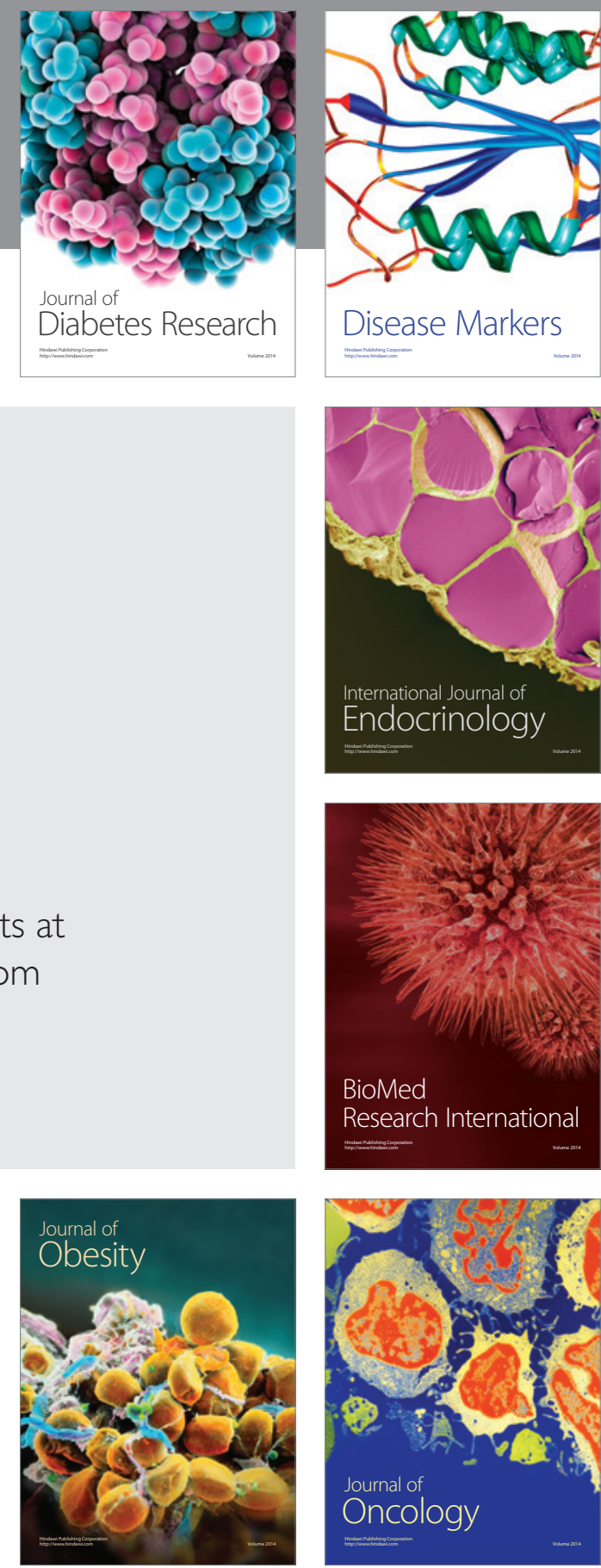

Disease Markers
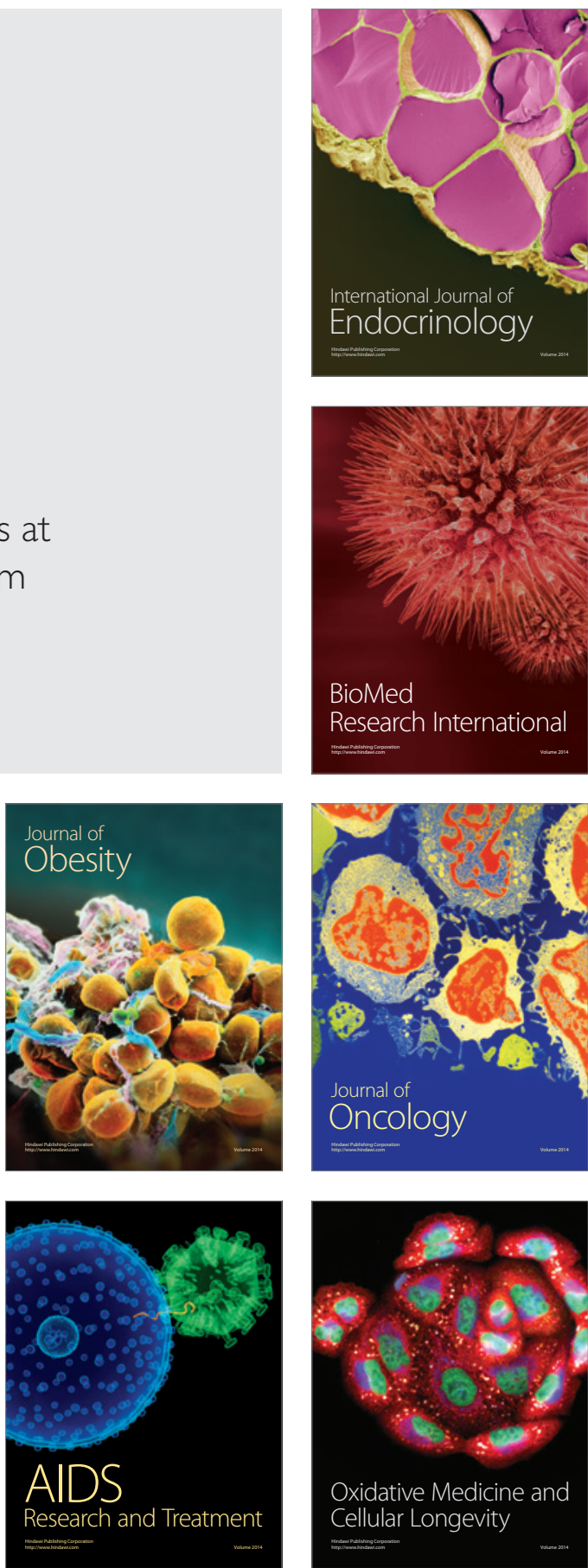